\title{
Generalized symmetric interpolating wavelets *
}

\author{
Zhuoer Shi ${ }^{\text {a }}$, Donald J. Kouri ${ }^{a}$, G.W. Wei ${ }^{b}$, David K. Hoffman ${ }^{c}$ \\ a Department of Physics, University of Houston, Houston, TX 77204-5506, USA \\ ${ }^{h}$ Department of Computational Science, National University of Singapore, 10 Kent Ridge Crescent, Singapore 119260. Singapore \\ c Department of Chemistry and Ames Laboratory, Iowa State University, Ames, IA 50011, USA
}

Received 16 November 1998

\begin{abstract}
A new class of biorthogonal wavelets-interpolating distributed approximating functional (DAF) wavelets are proposed as a powerful basis for scale-space functional analysis and approximation. The important advantage is that these wavelets can be designed with infinite smoothness in both time and frequency spaces, and have as well symmetric interpolating characteristics. Boundary adaptive wavelets can be implemented conveniently by simply shifting the window envelope. As examples, generalized Lagrange wavelets and generalized Sinc wavelets are presented and discussed in detail. Efficient applications in computational science and engineering are explored. (C) 1999 Elsevier Science B.V. All rights reserved.
\end{abstract}

PACS: 02.60

Keywords: Interpolating distributed approximating functional wavelets

\section{Introduction}

The theory of interpolating wavelets based on a subdivision scheme has attracted much attention recently $[1,9,12,13,17,22,27,29,40,42,45-49,54-56,65,66]$. Because the digital sampling space is exactly homomorphic to the multiscale spaces generated by interpolating wavelets, the wavelet coefficients can be obtained from linear combinations of discrete samples rather than from traditional inner product integrals. This parallel computational scheme significantly decreases the computational complexity and leads to an accurate wavelet decomposition, without any pre-conditioning or post-conditioning processes. Mathematically, various interpolating wavelets can be formulated in a biorthogonal setting.

Following Donoho's interpolating wavelet theory [12], Harten has described a kind of piecewise biorthogonal wavelet construction method [17]. Swelden independently develops this method as the well-known "lifting scheme" [56], which can be regarded as a special case of the Neville filters considered in [27]. The lifting scheme enables one to construct custom-designed biorthogonal wavelet transforms by just assuming a single low-pass filter (a smooth operation) without iterations. Theoretically, the interpolating wavelet theory is closely

\footnotetext{
* This work was supported by the National Science Foundation under Grant CHE-9700297, the R.A. Welch Foundation under Grant E-0608, Department of Energy under Contract 2-7405-ENG82, and the Energy Laboratory, University of Houston.
} 
related to the finite element technique in the numerical solution of partial differential equations, the subdivision scheme for interpolation and approximation, multi-grid generation and surface fitting techniques.

In this paper, we propose a new class of interpolating wavelets, which are generated from a generalized, window-modulated interpolating shell. Taking advantage of various interpolating shells, such as Lagrange polynomials and the Sinc function, etc., bell-shaped, smooth window modulation leads to wavelets with arbitrary smoothness in both time and frequency. Our method leads to a powerful and easily implemented series of interpolating wavelet. Generally, this novel designing technique can be extended to generate other non-interpolating multiresolution analyses as well (such as the Hermite shell). Unlike the biorthogonal solution discussed in [6], we do not attempt to solve a system of algebraic equations explicitly. We first choose an updating filter and then solve the approximation problem, which is a $r$ th-order accurate reconstruction of the discretization. Typically, the approximating functional is a piecewise polynomial. If we use the same reconstruction technique at all the points and at all levels of the dyadic sequence of uniform grids, the prediction will have a Toplitz-like structure.

These ideas are closely related to the distributed approximating functionals (DAFs) used successfully in computational chemistry and physics [20-22,65-67], for obtaining accurate, smooth analytical fits of potentialenergy surfaces in both quantum and classical dynamics calculations, as well as for the calculation of the state-to-state reaction probabilities for three-dimension (3-D) reactions. DAFs provide a numerical method for representing functions known only on a discrete grid of points. The underlying function or signal (image, communication, system, or human response to some probe, etc.) can be a digital time sequence (i.e., finite in length and 1-dimensional), a time and spatially varying digital sequence (including 2-D images that can vary with time, 3-D digital signals resulting from seismic measurements), etc. The general structure of the DAF representation of the function, $f_{\mathrm{DAF}}(x, t)$, where $x$ can be a vector (i.e.), not just a single variable, is

$$
\left.f_{\mathrm{DAF}}\left(x, t_{p}\right)=\sum_{j} \phi\left(x-x_{j}\right) \mid \sigma / \Delta\right) f\left(x_{j}, t_{p}\right),
$$

where $\left.\phi\left(x-x_{j}\right) \mid \sigma / \Delta\right)$ is the "discrete DAF", $f\left(x_{j}, t_{p}\right)$ is the digital value of the "signal" at time $t_{p}$, and $M$ and $\sigma / \delta$ will be specified in more detail below. They are adjustable DAF parameters, and for non-interpolative DAF, they enable one to vary the behavior of the above equation all the way from an interpolation limit, where

$$
f_{\mathrm{DAF}}\left(x_{j}, t_{p}\right) \equiv f\left(x_{j}, t_{p}\right)
$$

(i.e., the DAF simply reproduces the input data on the grid to as high accuracy as desired) to the well-tempered limit, where

$$
f_{\mathrm{DAF}}\left(x_{j}, t_{p}\right) \neq f\left(x_{j}, t_{p}\right)
$$

for function $f\left(x, t_{p}\right) \in L^{2}(R)$. Thus the well-tempered DAF does not exactly reproduce the input data. This price is paid so that instead a well-tempered DAF approximation makes the same order error off the grid as it does on the grid (i.e., there are no special points). We have recently shown that DAFs (both interpolating and non-interpolating) can be regarded as a set of scaling functionals that can be used to generate extremely robust wavelets and their associated biorthogonal complements, leading to a full multiresolution analysis $[22,46-$ $49,54,55,66,67]$. DAF-wavelets can therefore serve as an alternative basis for improved performance in signal and image processing.

The DAF wavelet approach can be applied directly to treat bounded domains. As shown below, the wavelet transform is adaptively adjusted around the boundaries of finite-length signals by conveniently shifting the modulated window. Thus the biorthogonal wavelets in the interval are obtained by using a one-sided stencil near the boundaries. Lagrange interpolation polynomials and band-limited Sinc functionals in Paley-Wiener space are two commonly used interpolating shells for signal approximation and smoothing, etc. Because of their importance in numerical analysis, we use these two kinds of interpolating shells to introduce our discussion. Other modulated windows, such as the square, triangle, B-spline and Gaussian are under study with regard to 
the time-frequency characteristics of generalized interpolating wavelets. By carefully designing the interpolating Lagrange and Sinc functionals, we can obtain smooth interpolating scaling functions with an arbitrary order of regularity.

\section{Interpolating wavelets}

The basic characteristics of interpolating wavelets of order $D$ discussed in Ref. [12] require that the primary scaling function, $\phi$, satisfies the following conditions:

(1) Interpolation:

$$
\phi(k)=\left\{\begin{array}{ll}
1, & k=0, \\
0, & k \neq 0,
\end{array} \quad k \in Z,\right.
$$

where $Z$ denotes the set of all integers.

(2) Self-Induced Two-Scale Relation: $\phi$ can be represented as a linear combination of dilates and translates of itself, with a weight given by the value of $\phi$ at $k / 2$,

$$
\phi(x)=\sum_{k} \phi(k / 2) \phi(2 x-k) .
$$

This relation is only approximately satisfied for some interpolating wavelets discussed in the later sections. However, the approximation can be made arbitrarily accurate.

(3) Polynomial Span: For an integer $D \geq 0$, the collection of formal sums symbol $\sum C_{k} \phi(x-k)$ contains all polynomials of degree $D$.

(4) Regularity: For a real $V>0, \phi$ is Hölder continuous of order $V$.

(5) Localization: $\phi$ and all its derivatives through order $\lfloor V\rfloor$ decay rapidly,

$$
\left|\phi^{(r)}(x)\right| \leq A_{s}(1+|x|)^{-s}, \quad x \in R, \quad s>0, \quad 0 \leq r \leq\lfloor V\rfloor,
$$

where $\lfloor V\rfloor$ represents the maximum integer that does not exceed $V$.

In contrast to most commonly used wavelet transforms, the interpolating wavelet transform possesses the following characteristics:

(1) The wavelet transform coefficients are generated by the linear combination of signal samplings,

$$
S_{j, k}=2^{-j / 2} f\left(2^{-i} k\right), \quad W_{j, k}=2^{-j / 2}\left[f\left(2^{-j}(k+1 / 2)\right)-\left(P_{j} f\right)\left(2^{-j}(k+1 / 2)\right)\right],
$$

instead of the convolution of the commonly used discrete wavelet transform, such as

$$
W_{j, k}=\int_{R} \psi_{j, k}(x) f(x) d x,
$$

where the scaling function, $\phi_{j, k}(x)=2^{j / 2} \phi\left(2^{j} x-k\right)$, and wavelet function, $\psi_{j, k}(x)=2^{j / 2} \psi\left(2^{j} x-k\right), P_{j} f$ as the interpolant $2^{-j / 2} \sum f\left(2^{-i} k\right) \phi_{j, k}(x)$.

(2) A parallel-computing mode can be easily implemented. The calculation and compression of coefficients does not depend on the results of other coefficients. For the halfband filter with length $N$ the calculation of each of the wavelet coefficients, $W_{j, k}$, does not exceed $N+2$ multiply/adds.

(3) For a $D$ th order differentiable function, the wavelet coefficients decay rapidly.

(4) In a minimax sense, threshold masking and quantization are nearly optimal approximations for a wide variety of regularity algorithms. 


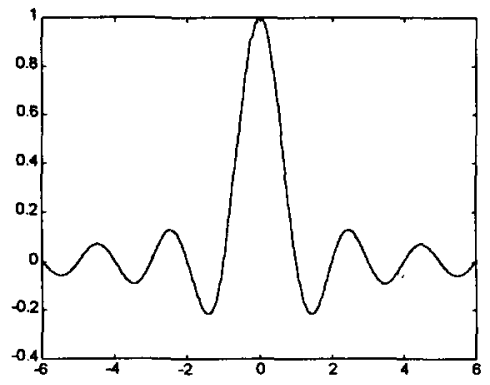

(a)

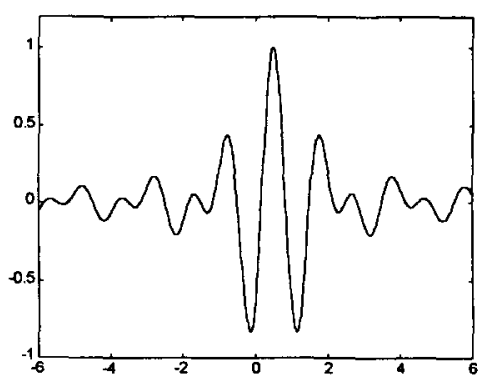

(b)

Fig. 1. $\pi$ band-limited interpolating wavelets. (a) Sinc function; (b) Sinclet wavelet.

Theoretically, interpolating wavelets are closely related to the following functions:

(1) Band-limited Shannon wavelets

The $\pi$ band-limited function, $\phi(x)=\sin (\pi x) /(\pi x) \in C^{\infty}$ in Paley-Wiener space, generates the interpolating functions. Every $\pi$ band-limited function $f \in L^{2}(R)$ can be reconstructed using the equation

$$
f(x)=\sum_{k} f(k) \frac{\sin \pi(x-k)}{\pi(x-k)},
$$

where the related wavelet function-Sinclet is defined as (see Fig. 1)

$$
\psi(x)=\frac{\sin \pi(2 x-1)-\sin \pi(x-1 / 2)}{\pi(x-1 / 2)} .
$$

(2) Interpolating fundamental splines

The fundamental polynomial spline of degree $D, \eta^{D}(x)$, where $D$ is an odd integer, has been shown by Schoenberg (1972) to be an interpolating wavelet (see Fig. 2). It is smooth with order $R=D-1$, and its derivatives through order $D-1$ decay exponentially [59]. Thus,

$$
\eta^{D}=\sum_{k} \alpha^{D}(k) \beta^{D}(x-k),
$$

where $\beta^{D}(x)$ is the $\mathrm{B}$-spline of order $D$ defined as

$$
\beta^{D}(x)=\sum_{j=0}^{D+1} \frac{(-1)^{j}}{D !}\left(\begin{array}{l}
D+1 \\
j
\end{array}\right)\left(x+\frac{D+1}{2}-j\right)^{D} U\left(x+\frac{D+1}{2}-j\right) .
$$

Here $U$ is the step function

$$
U(x)= \begin{cases}0, & x<0 \\ 1, & x \geq 0\end{cases}
$$

and $\left\{\alpha^{D}(k)\right\}$ is a sequence that satisfies the infinite summation condition

$$
\sum_{k} \alpha^{D}(k) \beta^{D}(n-k)=\delta(n) .
$$

(3) Deslauriers-Dubuc functional

Let $D$ be an odd integer, $D>0$. There exist functions $F_{D}$ such that if $F_{D}$ has already been defined at all binary rationals with denominator $2^{j}$, it can be extended by polynomial interpolation, to all binary rationals 


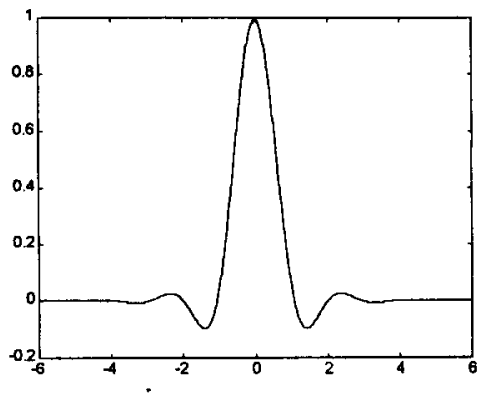

Fig. 2. Interpolating cardinal spline $(D=5)$.

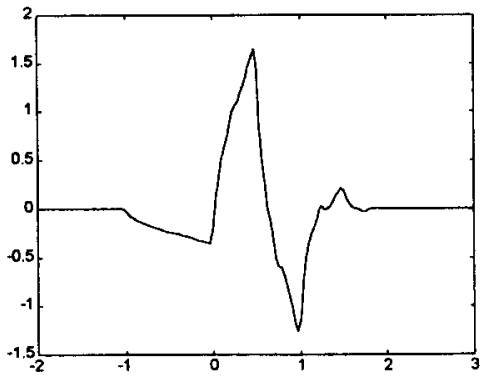

(a)

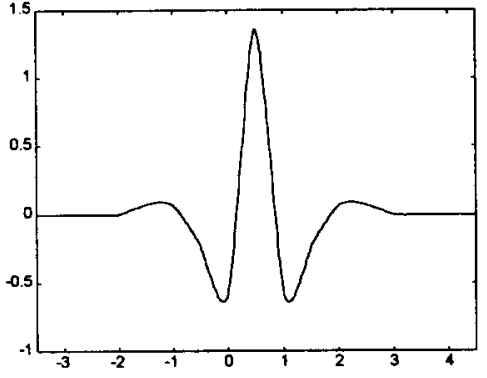

(b)

Fig. 3. Interpolating wavelets by auto-correlation shell $(D=3)$. (a) Daubechies wavelet; (b) Dubuc wavelet.

with denominator $2^{j+1}$, i.e. all points halfway between previously defined points $[9,13]$. Specifically, to define the function at $(k+1 / 2) / 2^{j}$ when it is already defined at all $\left\{k \times 2^{-j}\right\}$, fit a polynomial $\pi_{j, k}$ to the data $\left(k^{\prime} / 2^{j}, F_{D}\left(k^{\prime} / 2^{j}\right)\right.$ for $k^{\prime} \in\left\{2^{-j}[k-(D-1) / 2], \ldots, 2^{-j}[k+(D+1) / 2]\right\}$. This polynomial is unique,

$$
F_{D}\left(\frac{k+1 / 2}{2^{j}}\right) \equiv \pi_{j, k}\left(\frac{k+1 / 2}{2^{j}}\right)
$$

This subdivision scheme defines a function which is uniformly continuous at the rationals and has a unique continuous extension; $F_{D}$ is a compactly supported interval polynomial and is regular; it is the auto-correlation function of the Daubechies wavelet of order $D+1$. It is at least as smooth as the corresponding Daubechies wavelets (roughly twice as smooth).

(4) Auto-correlation shell of orthonormal wavelets

If $\breve{\phi}$ is an orthonormal scaling function, its auto-correlation $\phi=\breve{\phi} * \breve{\phi}(-\cdot)$ is an interpolating wavelet (Fig. 3) [40]. Its smoothness, localization and the two-scale relation are inherited from $\breve{\phi}$. The autocorrelations of Haar, Lamarie-Battle, Meyer, and Daubechies wavelets lead to, respectively, the interpolating Schauder, interpolating spline, $C^{\infty}$ interpolating, and Deslauriers-Dubuc wavelets.

(5) Lagrange half-band filters

Ansari, Guillemot, and Kaiser [1] used Lagrange symmetric halfband FIR filters to design the orthonomal wavelets that express the relation between the Lagrange interpolators and Daubechies wavelets [7]. Their filter corresponds to the Deslauriers-Dubuc wavelet of order $D=7(2 M-1), M=4$. The transfer function of the halfband symmetric filter $h$ is given by

$$
H(z)=\frac{1}{2}+z T\left(z^{2}\right),
$$


where $T$ is the trigonometric polynomial. Except for $h(O)=1 / 2$, at every even integer lattice point $h(2 n)=0, n \neq 0, n \in Z$. The transfer function of the symmetric FIR filter $h(n)=h(-n)$ has the form

$$
H(z)=1 / 2+\sum_{n=1}^{M} h(2 n-1)\left(z^{1-2 n}+z^{2 n-1}\right) .
$$

The concept of an interpolating wavelet decomposition is similar to "algorithm a trous", the connection having been found by Shensa [42]. The self-induced scaling and interpolation conditions are the most important characteristics of interpolating wavelets. From the following equation:

$$
f(x)=\sum_{n} f(n) \phi(x-n)
$$

and Eq. (1), the approximation to the signal is exact on the discrete sampling points, which does not hold in general for commonly used non-interpolating wavelets.

\section{Generalized interpolating wavelets}

In this paper, interpolating wavelets with either a Lagrange polynomial shell or Sinc functional shell are discussed in detail. We call these kinds of window modulated wavelets generalized interpolating wavelets, because they are more convenient to construct, processing and extend to higher dimensional spaces.

\subsection{Generalized Lagrange wavelets}

Three kinds of interpolating Lagrange wavelets, Halfband Lagrange wavelets, B-spline Lagrange wavelets and Gaussian-Lagrange DAF wavelets, are studied here as examples of the generalized interpolating wavelets.

Halfband Lagrange wavelets can be regarded as extensions of the Dubuc interpolating functionals [9,13], the auto-correlation shell wavelet analysis [40], and halfband filters [1]. B-spline Lagrange wavelets are generated by a B-spline-windowed Lagrange functional which increases the smoothness and localization properties of the simple Lagrange scaling function and its related wavelets. Lagrange Distributed Approximating Functionals (LDAF)-Gaussian modulated Lagrange polynomials, have been successfully applied for numerically solving various linear and nonlinear partial differential equations. Typical examples include DAF-simulations of 3dimensional reactive quantum scattering and the solution of a 2-dimensional Navier-Stokes equation with nonperiodic boundary conditions. In terms of a wavelet analysis, DAFs can be regarded as particular scaling functions (wavelet-DAFs) and the associated DAF-wavelets can be generated in a number of ways [20-22,65$67]$.

\subsubsection{Halfband Lagrange wavelets}

A special case of halfband filters can be obtained by choosing the filter coefficients according to the Lagrange interpolation formula. The filter coefficients are given by

$$
h(2 n-1)=\frac{(-1)^{n+M-1} \prod_{m=1}^{2 M}(M+1 / 2-m)}{(M-n) !(M+n-1) !(2 n-1)} .
$$

These filters have the property of maximal flatness in Fourier space, possessing a balance between the degree of flatness at zero frequency and the flatness at the Nyquist frequency (half sampling).

These half-band filters can be utilized to generate the interpolating wavelet decomposition, which can be regarded as a class of the auto-correlated shell of orthogonal wavelets, such as the Daubechies wavelets [7]. The 
interpolating wavelet transform can also be extended to higher order cases using different Lagrange polynomials, as [40]

$$
P_{2 n-1}(x)=\prod_{m=M+1, m \neq n}^{M} \frac{x-(2 m-1)}{(2 n-1)-(2 m-1)} .
$$

The predictive interpolation can be expressed as

$$
\Gamma S_{j}(i)=\sum_{n=1}^{M} P_{2 n-1}(0)\left[S_{j}(i+2 n-1)+S_{j}(i-2 n+1)\right], \quad i=2 k+1,
$$

where $\Gamma$ is a projection and $S_{j}$ is the $j$ th layer low-pass coefficients. This projection relation is equivalent to the subband filter response of

$$
h(2 n-1)=P_{2 n-1}(0) \text {. }
$$

The above-mentioned interpolating wavelets can be regarded as the extension of the fundamental DeslauriersDubuc interactive sub-division scheme, which results when $M=2$. The order of the Lagrange polynomial is $D=2 M-1=3$ (Fig. 6a).

It is easy to show that an increase of the Lagrange polynomial order $D$ will introduce higher regularity for the interpolating functionals (Fig. 7a). When $D \rightarrow+\infty$, the interpolating functional tends to a $\pi$ band-limited Sinc function and its domain of definition is on the real line. The subband filters generated by Lagrange interpolating functionals satisfy the properties:

(1) Interpolation: $h(\omega)+h(\omega+\pi)=1$.

(2) Symmetry: $h(\omega)=h(-\omega)$.

(3) Vanishing Moments: $\int_{R} x^{p} \phi(x) d x=\delta_{p}$.

Donoho outlines a basic subband extension to obtain a perfect reconstruction. He defines the wavelet function as

$$
\psi(x)=\phi(2 x-1) .
$$

The biorthogonal subband filters can be expressed as

$$
\tilde{h}(\omega)=1, \quad g(\omega)=e^{-i \omega}, \quad \tilde{g}(\omega)=e^{-i \omega} \overline{h(\omega)+\pi)} .
$$

However, the Donoho interpolating wavelets have some drawbacks. Because the low-pass coefficients are generated by a sampling operation only, as the decomposition layer increases, the correlation between low-pass coefficients become weaker. The interpolating (prediction) error (high-pass coefficients) strongly increases, which is deleterious to the efficient representation of the signal. Further, it cannot be used to generate a Riesz basis for $L^{2}(R)$ space.

Swelden has provided an efficient and robust scheme [56] for constructing biorthogonal wavelet filters. His approach can be utilized to generate high-order interpolating Lagrange wavelets with higher regularity. As Fig. 4 shows, $P_{0}$ is the interpolating prediction process, and the $P_{1}$ filter is called the updating filter, used to smooth the down-sampling low-pass coefficients. If we choose $P_{0}$ to be the same as $P_{1}$, then the new interpolating subband filters can be depicted as

$$
\left\{\begin{array}{l}
h_{1}(\omega)=h(\omega) \\
\tilde{h}(\omega)=1+\tilde{g}(\omega) \overline{P(2 \omega)}, \\
g_{1}(\omega)=e^{-i \omega}-h(\omega) P(2 \omega), \\
\tilde{g}_{1}(\omega)=\tilde{g}(\omega)
\end{array}\right.
$$




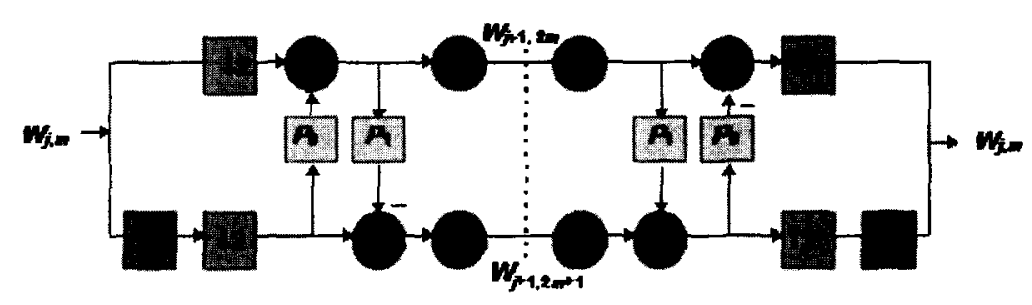

Fig. 4. Lifting scheme.

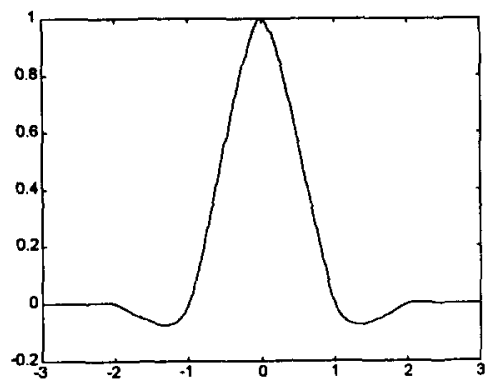

(a)

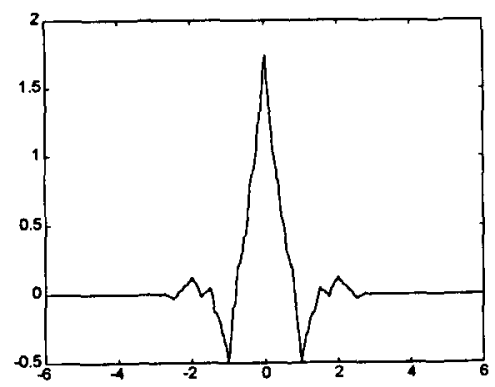

(c)

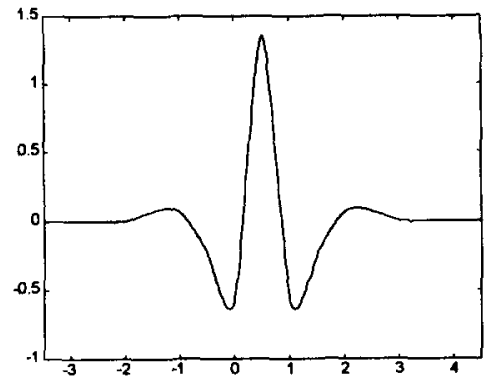

(b)

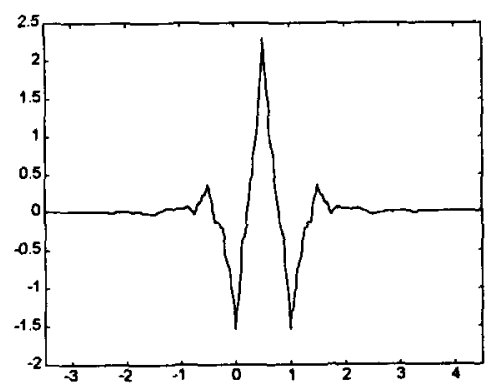

(d)

Fig. 5. Lagrange wavelets with $D=3$. (a) Scaling function; (b) Wavelet; (c) Dual scaling function; (d) Dual wavelet.

The newly developed filters $h_{1}, g_{1}, \tilde{h}_{1}$, and $\tilde{g}_{1}$ also generate the biorthogonal dual pair for a perfect reconstruction. Examples of biorthogonal lifting wavelets with regularity $D=3$ are shown in Fig. 5. Fig. 6 gives the corresponding Fourier responses of the equivalent subband decomposition filters.

\subsubsection{B-spline Lagrange wavelets}

Lagrange polynomials are natural interpolating expressions for functional approximations. Utilizing a different expression for the Lagrange polynomials, we can construct other forms of useful interpolating wavelets as follows.

We define a class of symmetric Lagrange interpolating functional shells as

$$
P_{M}(x)=\prod_{i=-M, i \neq 0}^{M} \frac{x-i}{-i} .
$$

It is easy to verify that this Lagrange shell also satisfies the interpolating condition on discrete, integer points, 


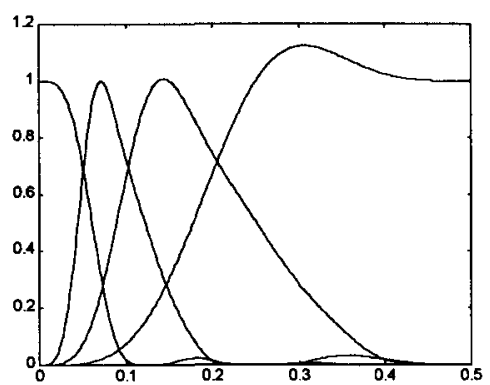

(a)

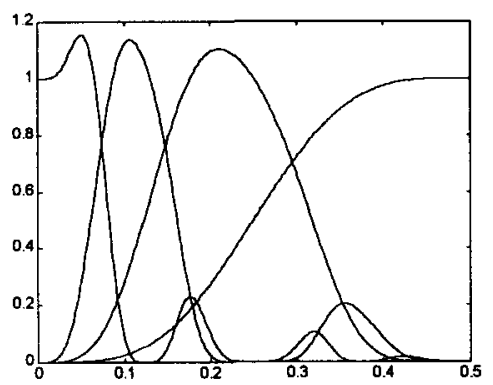

(b)

Fig. 6. Frequency response of equivalent filters $(D=3)$. (a) Decomposition; (b) Reconstruction.

$$
P_{M}(k)= \begin{cases}1, & k=0 \\ 0, & \text { otherwise } .\end{cases}
$$

However, simply defining the filter response as

$$
h(k)=P(k / 2) / 2, \quad k=-M, M,
$$

leads to nonstable interpolating wavelets, as shown in Fig. 7.

Including a smooth window, which vanishes at the zeros of the Lagrange polynomial, will lead to more regular interpolating wavelets and equivalent subband filters (as shown in Figs. 7 and 8). We select a welldefined B-spline function as the weight window. Then the scaling function (mother wavelet) can be defined as an interpolating B-spline Lagrange functional (BSLF),

$$
\begin{aligned}
\phi_{M}(x) & =\frac{\beta^{N}(x / \eta)}{\beta^{N}(0)} P_{M}(x) \\
& =\frac{\beta^{N}(x / \eta)}{\beta^{N}(0)} \prod_{i=M, i \neq 0}^{M} \frac{x-i}{-i},
\end{aligned}
$$

where $N$ is the B-spline order, and $\eta$ is the scaling factor to control the window width. To ensure coincidence of the zeroes of the B-spline and the Lagrange polynomial, we set

$$
2 M=\eta \times(N+1) .
$$

To ensure the interpolation condition, the B-spline envelope degree $M$ must be an odd number. It is easy to show that if the B-spline order is $N=4 k+1, \eta$ can be any odd integer $(2 k+1)$; if $N$ is an even integer, then $\eta$ can only be 2 . When $N=4 k-1$, we cannot construct an interpolating shell using the definition above. From the interpolation and self-induced scaling properties of the interpolating wavelets, it is easy to verify that

$$
h(k)=\phi_{M}(k / 2) / 2, \quad k=-2 M+1, \quad 2 M-1 .
$$

\subsubsection{Gaussian-Lagrange DAF wavelets}

We can also select a class of distributed approximation functional-Gaussian-Lagrange (GLDAF) as our basic scaling function to construct interpolating wavelets. We write

$$
\phi_{M}(x)=W_{\sigma}(x) P_{M}(x)
$$




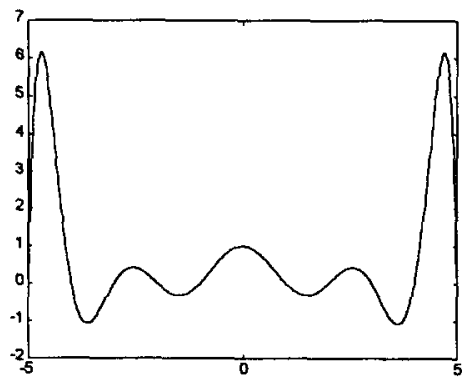

(a)

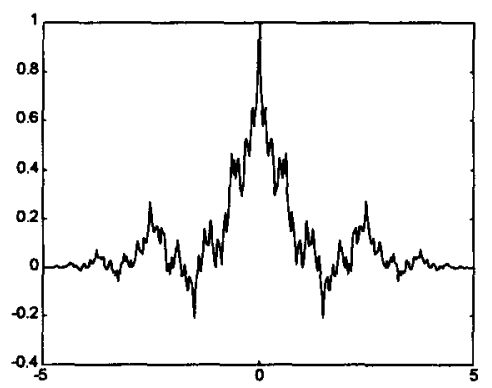

(b)

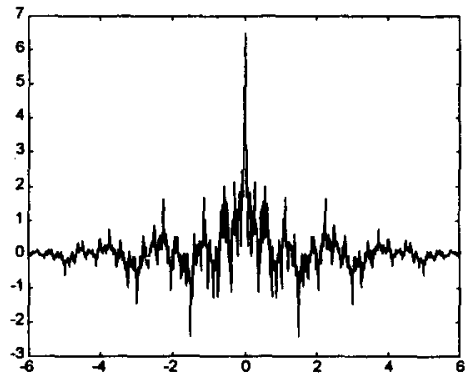

(d)

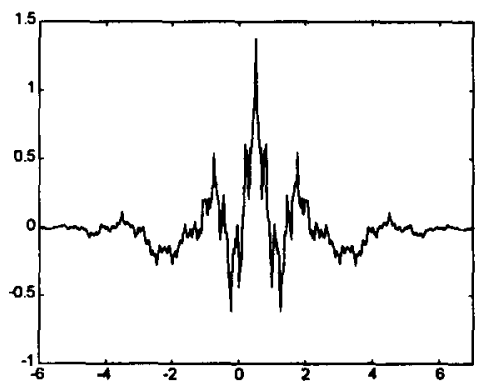

(c)

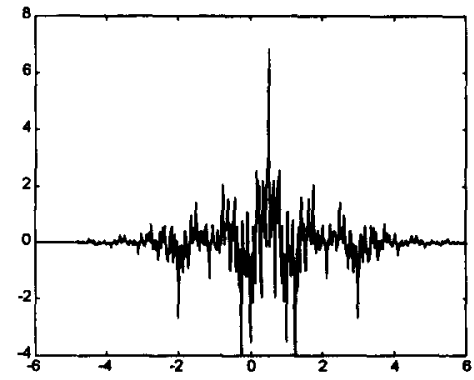

(e)

Fig. 7. Nonregularized Lagrange wavelets $(M=5)$. (a) Lagrange polynomial; (b) Scaling function; (c) Wavelet; (d) Dual scaling function; (e) Dual wavelet.

$$
=W_{\sigma}(x) \prod_{i=-M, i \neq 0}^{M} \frac{x-i}{-i},
$$

where $W_{\sigma}(x)$ is a window function. It is chosen to be a Gaussian,

$$
W_{\sigma}(x)=e^{-x^{2} / 2 \sigma^{2}}
$$

because it satisfies the minimum frame bound condition in quantum physics. Here $\sigma$ is a window width parameter, and $P_{M}(x)$ is the Lagrange interpolation kernel. The DAF scaling function has been successfully introduced as an efficient and powerful grid method for quantum dynamical propagations [40]. Using Swelden's lifting scheme [32], a wavelet basis is generated. The Gaussian window in our DAF-wavelets efficiently smoothes out the Gibbs oscillations, which plague most conventional wavelet bases. The following equation shows the connection between the B-spline window function and the Gaussian window [34]: 


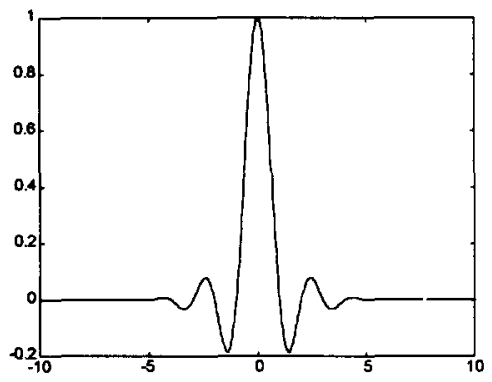

(a)

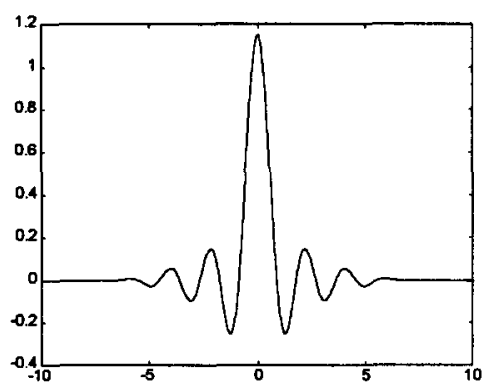

(c)

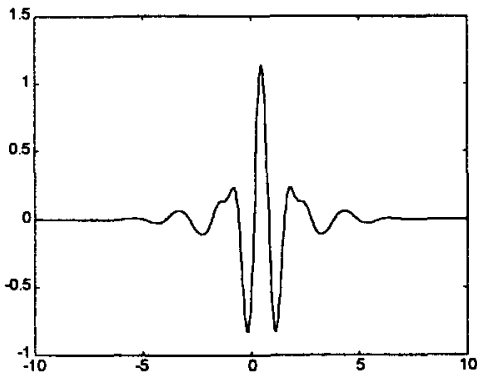

(b)

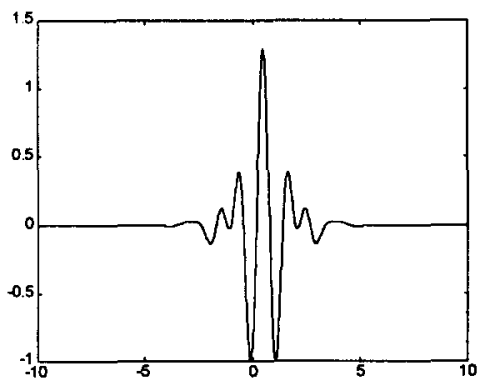

(d)

Fig. 8. B-spline Lagrange DAF wavelets $(N=4, \eta=2)$. (a) Scaling function; (b) Wavelet; (c) Dual scaling function; (d) Dual wavelet.

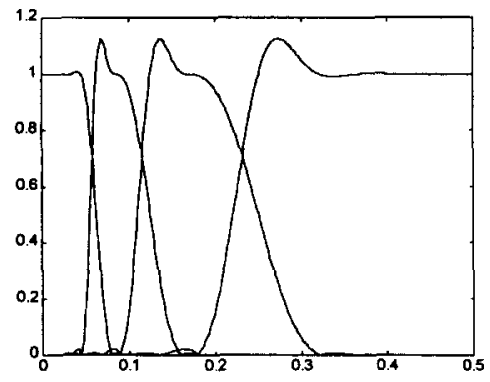

(a)

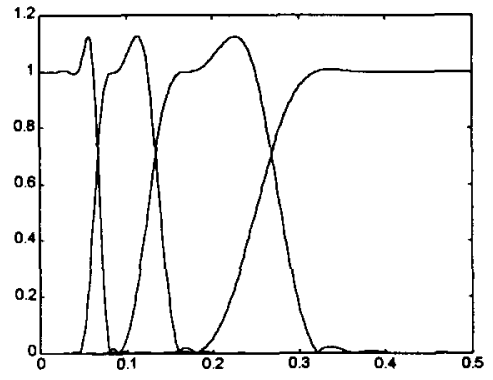

(b)

Fig. 9. Frequency response of equivalent filters $(N=4, \eta=2)$. (a) Decomposition; (b) Reconstruction.

$$
\beta^{N}(x) \cong \sqrt{\frac{6}{\pi(N+1)}} \exp \left(\frac{-6 x^{2}}{N+1}\right),
$$

for large $N$. As in Fig. 12, if we choose the window width

$$
\sigma=\eta \sqrt{(N+1) / 12},
$$

the Gaussian-Lagrange wavelets generated by the lifting scheme will be similar to the B-spline Lagrange wavelets. Usually, the Gaussian-Lagrange DAF displays a slightly better smoothness and more rapid decay than the B-spline Lagrange wavelets. If we select more sophisticated window shapes, such as those popular in engineering (Bartlett, Hanning, Hamming, Blackman, Chebychev, and Bessel windows), the Lagrange wavelets can be generalized further. We shall call these extensions Bell-windowed Lagrange wavelets. 


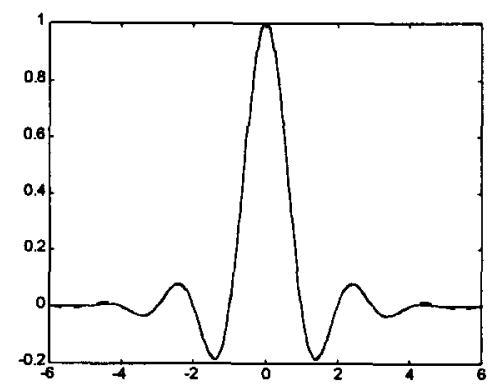

Fig. 10. Mother wavelet comparison $(N=4, \eta=2)$. Solid: B-spline Lagrange: dotted: Gaussian-Lagrange.

\subsection{Generalized Sinc wavelets}

As we have mentioned above, the $\pi$ band-limited Sinc function,

$$
\phi(x)=\sin (\pi x) /(\pi x) \in C^{\infty},
$$

in Paley-Wiener space constructs an interpolating function. Every $\pi$ band-limited function $f \in L^{2}(R)$ can be reconstructed by the equation

$$
f(x)=\sum_{k} f(k) \frac{\sin \pi(x-k)}{\pi(x-k)},
$$

where the related wavelet function-Sinclet is defined as (see Fig. 1)

$$
\phi(x)=\frac{\sin \pi(2 x-1)-\sin \pi(x-1 / 2)}{\pi(x-1 / 2)} .
$$

The scaling Sinc function is the well-known ideal low-pass filter, which possesses the ideal square filter response as

$$
H(\omega)= \begin{cases}1, & |\omega| \leq \pi / 2 \\ 0, & \pi / 2<|\omega| \leq \pi\end{cases}
$$

Its impulse response can be generated as

$$
h[k]=\int_{(-\pi / 2, \pi / 2)} e^{i k \omega} d \omega / 2 \pi=\sin (\pi k / 2) / \pi k .
$$

The so-called half-band filter possesses a nonzero impulse only at the odd integer sampler, $h(2 k+1)$, while at even integers, $h[2 k]=0$ unless a $k=0$.

However, this ideal low-pass filter is never used in application. Since the digital filter is an IIR (infinite impulse response) solution, its use as a digital cutoff FIR (finite impulse response) will produce Gibbs phenomenon (overshot effect) in Fourier space, which degrades the frequency resolution (Fig. 11).

The resulting compactly supported Sinc scaling and wavelet functions, as well as their biorthogonal dual scaling and wavelet functions, are shown in Fig. 12. We see that the regularity of the cutoff Sinc is obviously degraded with a fractal-like shape, which leads to poor time localization.

\subsubsection{B-spline Sinc wavelets}

Because the ideal low-pass Sinc wavelet cannot be implemented "ideally" by FIR (finite impulse response) filters, to eliminate the cutoff singularity, a windowed weighting technique is employed to adjust the timefrequency localization of the Sinc wavelet analysis. 


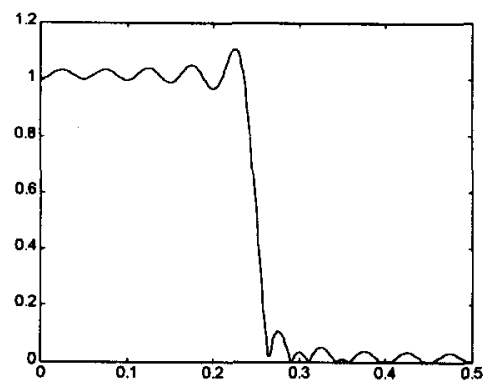

Fig. 11. Gibbs overshoot of the Sinc FIR.

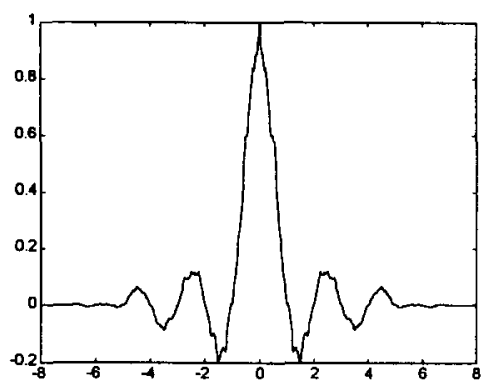

(a)

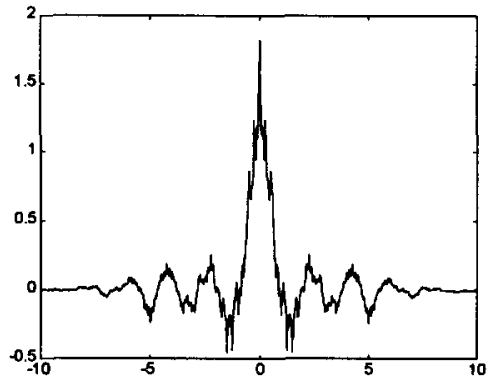

(c)

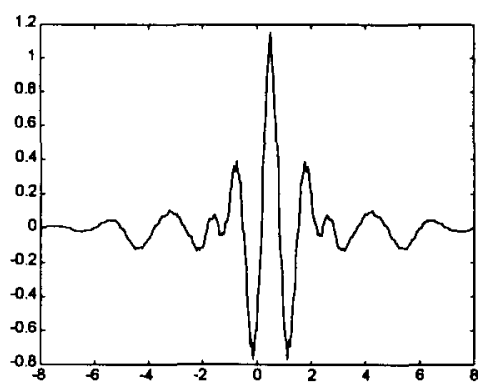

(b)

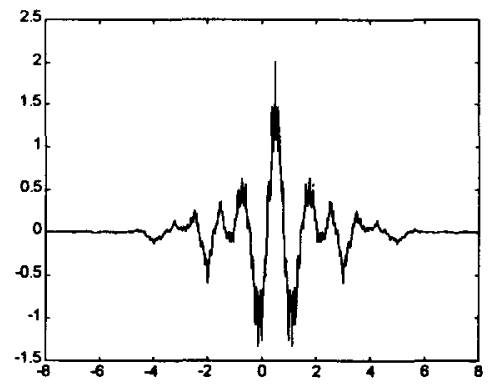

(d)

Fig. 12. Sinc cutoff wavelets $(M=9)$. (a) Scaling; (b) Wavelet; (c) Dual scaling; (d) Dual wavelet.

To begin, we define a symmetric Sinc interpolating functional shell as

$$
P(x)=\frac{\sin (\pi x / 2)}{\pi x} .
$$

Utilizing a smooth window, which vanishes gradually at the exact zeros of the Sinc functional, will lead to more regular interpolating wavelets and equivalent subband filters (as shown in Figs. 13 and 14).

For example, we illustrate using a well-defined B-spline function as the weight window. Then the scaling function (mother wavelet) can be defined as an interpolating B-spline Sinc functional (BSF),

$$
\phi_{M}(x)=\frac{\beta^{N}(x / \eta)}{\beta^{N}(0)} P(x)
$$




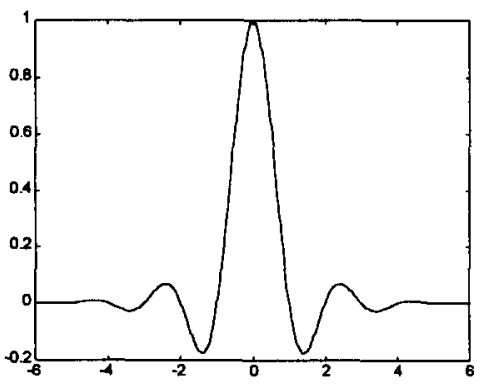

(a)

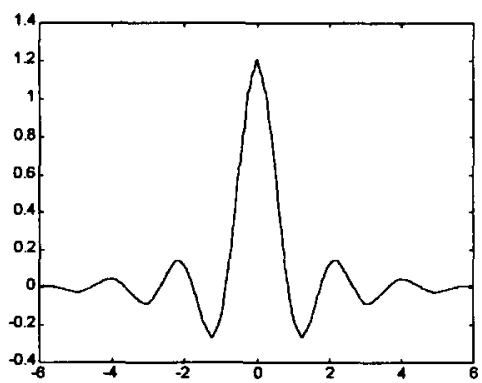

(c)

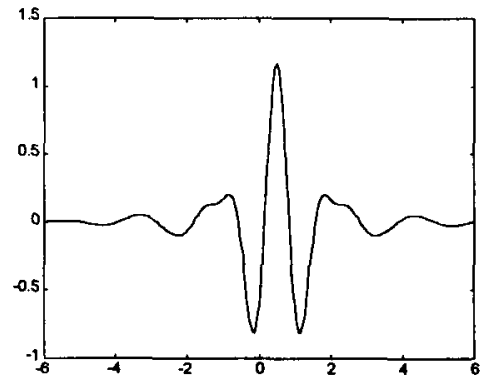

(b)

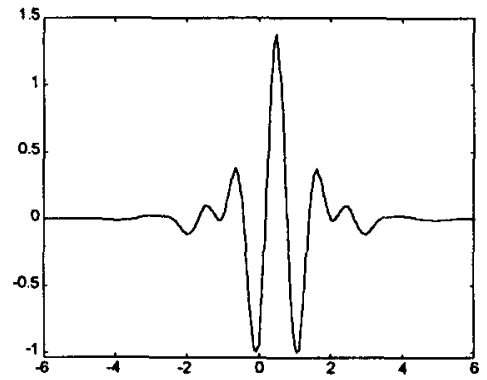

(d)

Fig. 13. B-spline Lagrange DAF wavelets $(N=5, \eta=3$ ). (a) Scaling; (b) Wavelet; (c) Dual scaling; (d) Dual wavelet.

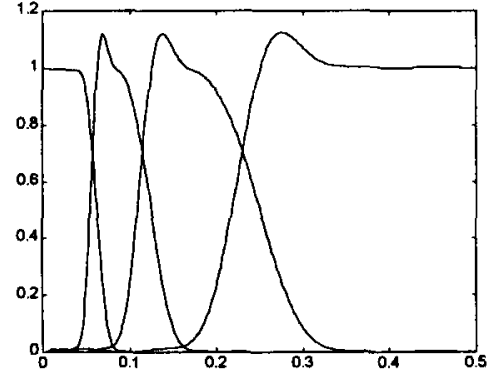

(a)

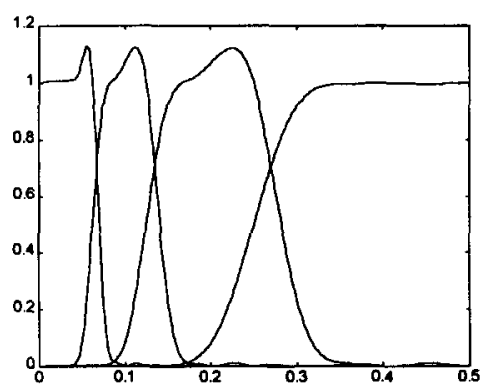

(b)

Fig. 14. Frequency response of equivalent filters $(N=5, \eta=3)$. (a) Decomposition; (b) Reconstruction.

$$
=\frac{\beta^{N}(x / \eta)}{\beta^{N}(0)} \frac{\sin (\pi x / 2)}{\pi x},
$$

where $N$ is the $\mathrm{B}$-spline order, and $\eta$ is the scaling factor to control the window width. To ensure the coincidence of the zeroes of the B-spline and the Sinc shell, we set

$$
2 M+1=\eta \times(N+1) / 2 .
$$

To maintain the interpolation condition, $h(2 k)=0, k \neq 0$, it is easy to show that when the B-spline order $N=4 k+1, \eta$ may be any odd integer $(2 k+1)$. If $N$ is an even integer, then $\eta$ can only be 2 . When $N=4 k-1$, we cannot construct the interpolating shell using the definition above. The admissibility condition can be expressed as 


$$
\begin{cases}\eta=2, & N=2 i \\ \eta=2 k+1, & N=4 i+1\end{cases}
$$

From the interpolation relation

$$
\phi(k)=\left\{\begin{array}{ll}
1, & k=0, \\
0, & k \neq 0,
\end{array} \quad k \in Z,\right.
$$

and the self-induced two-scale relation

$$
\phi(x)=\sum \phi(k / 2) \phi(2 x-k),
$$

it is easy to show that

$$
h(k)=\phi_{M}(k / 2) / 2, \quad k=-2 M+1, \quad 2 M-1 .
$$

\subsubsection{Gaussian-Sinc DAF wavelets}

We can also select a class of distributed approximation functionals, i.e., the Gaussian-Sinc DAF (GSDAF) as our basic scaling function to construct interpolating scalings,

$$
\begin{aligned}
\phi_{M}(x) & =W_{\sigma}(x) P(x) \\
& =W_{\sigma}(x) \frac{\sin (\pi x / 2)}{\pi x},
\end{aligned}
$$

where $W_{\sigma}(x)$ is a window function which is selected as a Gaussian,

$$
W_{\sigma}(x)=e^{-x^{2} / 2 \sigma^{2}} .
$$

Because it satisfies the minimum frame bound condition in quantum physics, it significantly improves the time-frequency resolution of the Windowed-Sinc wavelet. Here $\sigma$ is a window width parameter, and $P(x)$ is the Sinc interpolation kernel. This DAF scaling function has been successfully used in an efficient and powerful grid method for quantum dynamical propagations [40]. Moreover, the Hermite DAF is known to be extremely accurate for solving the 2-D harmonic oscillator, for calculating the eigenfunctions and eigenvalues of the Schrödinger equation. The Gaussian window in our DAF-wavelets efficiently smoothes out the Gibbs oscillations, which plague most conventional wavelet bases. The following equation shows the connection between the B-spline and the Gaussian windows [34]:

$$
\beta^{N}(x) \cong \sqrt{\frac{6}{\pi(N+1)}} \exp \left(\frac{-6 x^{2}}{N+1}\right),
$$

for large $N$. As in Fig. 6, if we choose the window width

$$
\sigma=\eta \sqrt{(N+1) / 12},
$$

the Gaussian Sinc wavelets generated by the lifting scheme will be similar to the B-spline Sinc wavelets. Usually, the Gaussian Sinc DAF displays a slightly better smoothness and rapid decay than the B-spline Lagrange wavelets. If we select more sophisticated window shapes, the Sinc wavelets can be generalized further. We call these extensions Bell-windowed Sinc wavelets. The available choices can be any of the popularly used DFT (discrete Fourier transform) windows, such as Bartlett, Hanning, Hamming, Blackman, Chebychev, and Besel windows. 


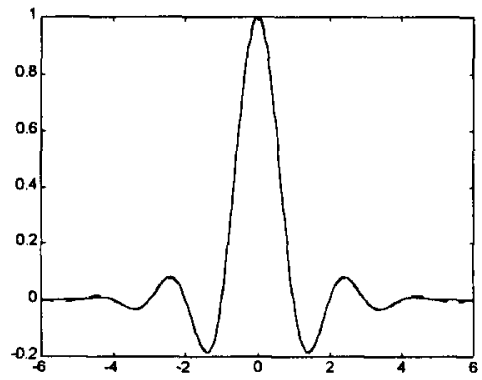

Fig. 15. Mother wavelet comparison $(N=4, \eta=2)$. Solid: B-spline Sinc; dotted: Gaussian Sinc.

\section{Adaptive boundary adjustment}

The above-mentioned generalized interpolating wavelet is defined on the domain $C(R)$. Many engineering applications involve finite length signals, such as image and isolated speech segments. In general, we can define these signals on $C[0,1]$. One could set the signal equal to zero outside [0,1], but this introduces an artificial "jump" discontinuity at the boundaries, which is reflected in the wavelet coefficients. It will degrade the signal filtering and compression in multiscale space. Developing wavelets adapted to "life on an interval" is useful. Periodization and symmetric periodization are two commonly used methods to reduce the effect of edges. However, unless the finite length signal has a large flat area around the boundaries, these two methods cannot remove the discontinuous effects completely $[4,6,11]$. Dubuc utilized an iterative interpolating function, $F_{D}$, on the finite interval to generate an interpolation on the set of dyadic rationals $D_{j}$. The interpolation in the neighborhood of the boundaries is treated using a boundary-adjusted functional, which leads to regularity of the same order as in the interval. This avoids the discontinuity that results from periodization or extending by zero. It is well known that this results in weaker edge effects, and that no extra wavelet coefficients (to deal with the boundary) have to be introduced, provided the filters used are symmetric.

We let $K_{j}$ represent the number of coefficients at resolution layer $j$, where $K_{j}=2^{j}$. Let $2^{j}>2 D+2$, define the non-interacting decomposition. If we let $j_{0}$ hold the non-interaction case $2^{j 0}>2 D+2$, then there exist functions $\phi_{j, k}^{\text {Interval }}, \psi_{j, k}^{\text {Interval }}$ such that for all $f \in C[0,1]$,

$$
f=\sum_{k=0}^{2^{j_{0}}-1} S_{j_{0}}(k) \phi_{j_{0}, k}^{\text {Interval }}+\sum_{j \geq j_{0}} \sum_{k=0}^{2^{j}-1} W_{j}(k) \psi_{j, k}^{\text {Interval }} .
$$

The $\phi_{j, k}^{\text {Interval }}, \psi_{j, k}^{\text {Interval }}$ are called the interval interpolating scalings and wavelets, which satisfy the interpolation conditions

$$
\begin{cases}\phi_{j, k}^{\text {Interval }}\left(2^{-j} n\right)=2^{j / 2} \delta_{k, n}, & 0 \leq n<K_{j}, \\ \psi_{j, k}^{\text {Interval }}\left(2^{-j-1} n\right)=2^{j / 2} \delta_{2 k+1, n}, & 0 \leq n<K_{j+1} .\end{cases}
$$

The interval scaling is defined as

$$
\phi_{j, k}^{\text {Interval }}= \begin{cases}\phi_{j, k}^{\text {Left }}, & 0 \leq k \leq D, \\ \left.\phi_{j, k}\right|_{[0,1]}, & D<k<2^{j}-D-1, \\ \phi_{j, k}^{\text {Right }}, & 2^{j}-D-1 \leq k<2^{j},\end{cases}
$$

where $\left.\phi_{j, k}\right|_{\{0,1\}}$ is called the "inner-scaling" which is identical to the fundamental interpolating function, and $\phi_{j, k}^{\text {Right }}$ and $\phi_{j, k}^{\text {Left }}$ are the "left-boundary" and the "right-boundary" scalings, respectively. Both are as smooth as 
$\left.\phi_{j, k}\right|_{[0,1]}$. Interval wavelets are defined as

$$
\psi_{j, k}^{\text {Interval }}= \begin{cases}\psi_{j, k}^{\text {Left }}, & 0 \leq k<\lfloor D / 2\rfloor, \\ \left.\psi_{j, k}\right|_{[0,1]}, & \lfloor D / 2\rfloor \leq k<2^{j}-\lfloor D / 2\rfloor, \\ \psi_{j, k}^{\text {Right }}, & 2^{j}-\lfloor D / 2\rfloor \leq k<2^{j} .\end{cases}
$$

$\left.\psi_{j, k}\right|_{[0,1]}$ is the inner-wavelet, and $\psi_{j, k}^{\text {Left }}$ and $\psi_{j, k}^{\text {Right }}$ are the left and right-boundary wavelets, respectively, which are of the same order regularity as the inner-wavelet [13].

The corresponding factors for the Deslauriers-Dubuc interpolating wavelets are $M=2$, and the order of the Lagrange polynomial is $D=2 M-1=3$. The interpolating wavelet transform can be extended to high order cases by two kinds of Lagrange polynomials, where the inner-polynomials are defined as [14]

$$
P_{2 n-1}(x)=\prod_{m=-M+1, m \neq n} \frac{x-(2 m-1)}{(2 n-1)-(2 m-1)} .
$$

This kind of polynomial introduces the interpolation in the intervals according to

$$
P_{j} S(i)=\sum_{n=1}^{M} P_{2 n-1}(0)\left[S_{j}(i+2 n-1)+S_{j}(i-2 n+1)\right], \quad i=2 k+1,
$$

and the boundary polynomials are

$$
L_{d}(x)=\prod_{m=0, m \neq d}^{2 M-1} \frac{x-m}{d-m}, \quad 0 \leq d \leq D,
$$

which introduce the adjusted interpolation on the two boundaries of the intervals. That is,

$$
P_{j} S(i)=\sum_{d=0}^{D} L_{d}(i / 2) S_{j}(i+2 d-1), \quad i=2 k+1, \quad 0 \leq k \leq\lfloor D-1\rfloor / 2 .
$$

The left boundary extrapolation outside the intervals is defined as

$$
P_{j} S(-1)=\sum_{d=0}^{D} L_{d}(-1 / 2) S_{j}(2 d), \quad i=2 k+1, \quad 0 \leq k \leq\lfloor D-1\rfloor / 2,
$$

and the right boundary extrapolation is similar to the above. The boundary adjusted interpolating scaling is shown in Fig. 16.

Although Dubuc shows the interpolation is almost twice differentiable, there still is a discontinuity in the derivative. In this paper, a DAF-wavelet based boundary adjusted algorithm is introduced. This technique can produce an arbitrary smooth derivative approximation, because of the infinitely differentiable character of the Gaussian envelope. The boundary-adjusted scaling functionals are generated as conveniently as possible just by window shifting and satisfy the following equation:

$$
\phi_{m}(x)=W(x-2 m) P(x), \quad m=-\lfloor(M-1) / 2\rfloor,\lfloor(M-1) / 2\rfloor,
$$

where $\phi_{m}(x)$ represents different boundary scalings, $W(x)$ is the generalized window function and $P(x)$ is the symmetric interpolating shell. When $m>0$, left boundary functionals are generated; when $m<0$, we obtain right boundary functionals. The case $m=0$ represents the inner scalings mentioned above. One example for 


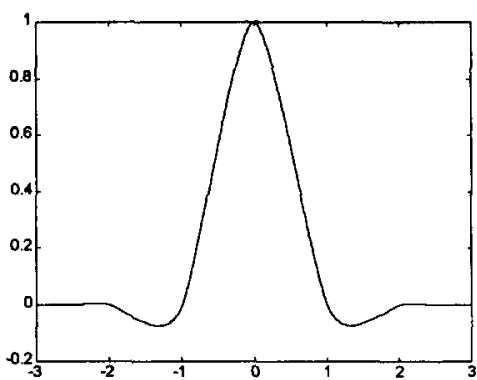

(a)

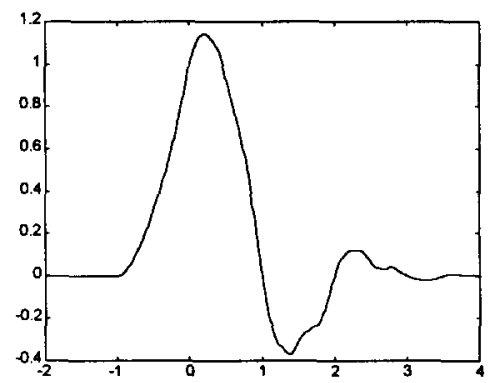

(b)

Fig. 16. Dubuc wavelets $(D=3)$. (a) Inner scaling; (b) Boundary scaling.

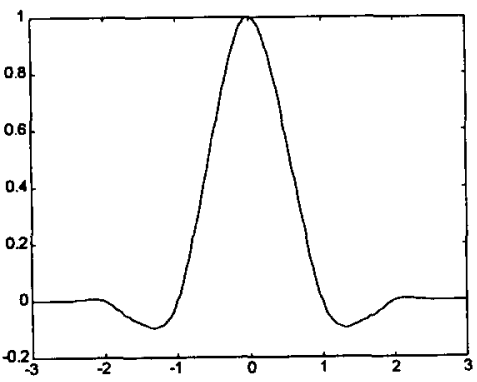

(a)

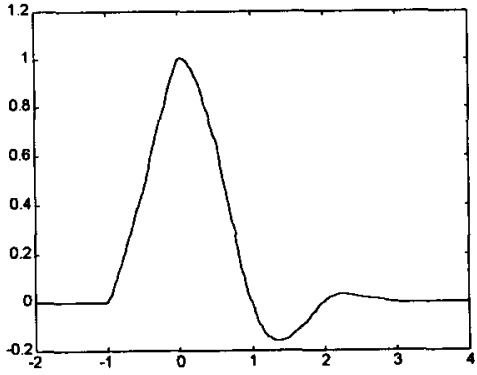

(b)

Fig. 17. Example for a Sinc-DAF wavelet.

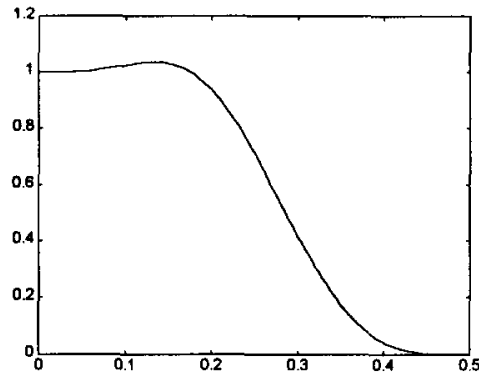

(a)

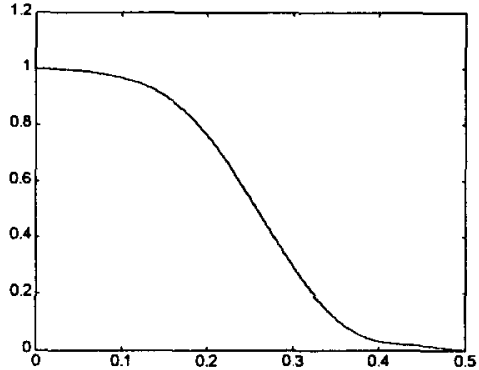

(b)

Fig. 18. Boundary filter response comparison between the halfband Lagrange wavelet and our DAF wavelet.

a Sinc-DAF wavelet is shown in Fig. 17. We choose the compactly-supported length of the scaling function to be the same as the halfband Lagrange wavelet. It is easy to show that our newly developed boundary scaling is smoother than the commonly used Dubuc boundary interpolating functional. Thus it will generate a more stable boundary adjusted representation for finite-length wavelet transforms, as well as a better derivative approximation around the boundaries. Fig. 18 is the boundary filter response comparison between the halfband Lagrange wavelet and our DAF wavelet. It is easy to establish that our boundary response decreases the overshoot of the low-pass band filter, and so is more stable for boundary frequency analysis and approximation. 
Table 1

Eigenvalues of the 2D harmonic oscillator

\begin{tabular}{|c|c|c|c|}
\hline$k=k_{x}+k_{y}$ & $k_{d}$ & Exact solution & Sinc-DAF calculation \\
\hline 0 & 1 & 1 & 0.99999999999835 \\
\hline 1 & 2 & 2 & $\begin{array}{l}1.99999999999952 \\
1.99999999999965\end{array}$ \\
\hline 2 & 3 & 3 & $\begin{array}{l}2.99999999999896 \\
2.99999999999838 \\
2.99999999999997\end{array}$ \\
\hline 3 & 4 & 4 & $\begin{array}{l}3.99999999999943 \\
3.99999999999947 \\
3.99999999999986 \\
3.99999999999994\end{array}$ \\
\hline 4 & 5 & 5 & $\begin{array}{l}4.99999999999907 \\
4.99999999999953 \\
4.99999999999989 \\
5.00000000000674 \\
5.00000000000813\end{array}$ \\
\hline 6 & 6 & 6 & $\begin{array}{l}5.99999999999982 \\
6.00000000000018 \\
6.00000000000752 \\
6.00000000000801 \\
6.00000000044972 \\
6.00000000012005\end{array}$ \\
\hline
\end{tabular}

\section{Applications of generalized symmetric interpolating wavelets}

\subsection{Eigenvalue solution of $2 D$ quantum harmonic oscillator}

As discussed in [22], a standard eigenvalue problem of the Schrödinger equation is that of the 2D harmonic oscillator,

$$
\left[-\frac{h^{2}}{2 m} \sum_{i=1}^{2} \frac{\partial^{2}}{\partial x_{i}^{2}}+\frac{1}{2}\left(x_{1}^{2}+x_{2}^{2}\right)\right] \Phi_{k}\left(x_{1}, x_{2}\right)=E_{k} \Phi_{k}\left(x_{1}, x_{2}\right) .
$$

Here $\Phi_{k}$ and $E_{k}$ are the $k$ th eigenfunction and eigenvalue, respectively. The eigenvalues are given exactly by

$$
E_{k_{1}, k_{2}}=1+k_{1}+k_{2}, \quad 0 \leq k<\infty, \quad 0 \leq k_{1} \leq k_{2},
$$

with a degeneracy $\left(k_{d}=k+1\right)$ in each energy level $E_{k}=1+k$. The 2D version of the wavelet DAF representation of the Hamiltonian operator was constructed and the first 21 eigenvalues and eigenfunctions obtained by subsequent numerical diagonalization of the discrete Sinc-DAF Hamiltonian. As shown in Table 1 all results are accurate to at least 10 significant figures for the first 16 eigenstates. It is evident that DAF-wavelets are powerful for solving eigenvalue problems.

\subsection{Target extraction}

Military target extraction, including such applications as high-resolution radar (aerial photograph) imaging, radar echo, and remote sense detection, is a difficult subject. National Defense Agencies (e.g., the Navy, 


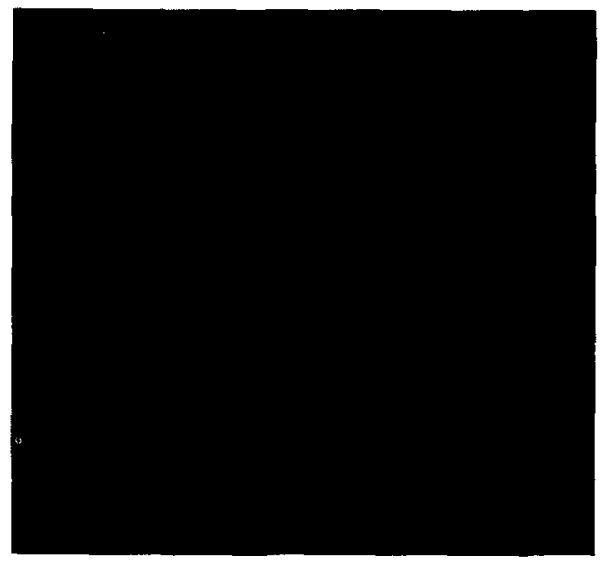

(a)

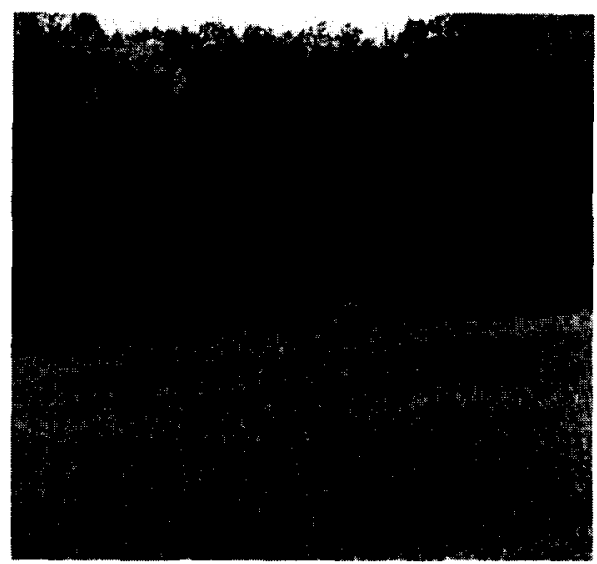

(b)

Fig. 19. Target extraction from color background. (a) Original pilot view; (b) DAF-wavelet restoration.

Army, and Air Force) have great interest in technical advances for reconnaissance, earlier warning and target recognition. Some of our DAF-wavelet schemes represent a significant breakthrough for these very difficult tasks. Compared with other methods, our algorithm possesses a very high resolution for target localization and high efficiency for clutter/noise suppression, as well as computational efficiency.

Detecting a military target in a low luminance environment is challenging work for image processing. To improve the target discrimination, the visibility of differences between a pair of images is important for modern image restoration and enhancement. We construct a method for detectability using a multiple channel enhancement technique. The images were captured on a color monitor at a viewing distance giving 95 pixels per degree of visual angle and an image size of $5.33 \times 5.05 \mathrm{deg}$. The mean luminance of the images was about $10 \mathrm{~cd} / \mathrm{m}^{2}$ [39]. Using our newly developed visual enhancement techniques, visual targets can be extracted very accurately in a low-luminance environment for detection and warning. The technique combines the response of human vision system (HVS) with multiresolution enhancement and restoration methods. The simulation of tank-target detection in a low-luminance environment is shown in Fig. 19.

\subsection{Image filtering}

Image de-noising is a difficult problem for signal processing. Due to the complicated structure of image and background noise, an optimal filtering technique does not currently exist. Generally, the possible noise sources include photoelectric exchange, photo spots, image communication error, etc. Such noise causes the visual perception to generate speckles, blips, ripples, bumps, ringing and aliasing. The noise distortion not only affects the visual quality of images, but also degrades the efficiency of data compression and coding. De-noising and smoothing are extremely important for image processing.

We use a DAF-wavelet to generate a more efficient, human-vision-system-based image processing technique, which processes the advantages of (1) long range de-correlation for convenience of compression and filtering; (2) high perceptual sensitivity and robustness; (3) filtering that takes account of the human visual response. It therefore can enhance the most important visual information, such as edges, while suppressing the large scale of flat regions and background; (4) it can be carried out with real-time processing.

Biorthogonal interpolating wavelets and corresponding filters are constructed based on Gauss-Lagrange distributed approximating functionals (DAFs). The utility of these DAF wavelets and filters is tested for digital image de-noising in combination with a novel blind restoration technique. This takes account of the response of human vision system so as to remove the perceptual redundancy and obtain better visual performance in image 


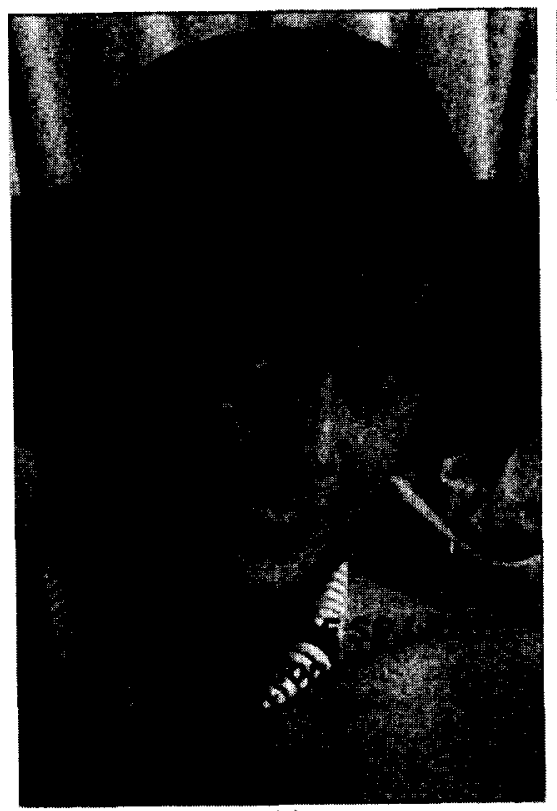

(a)

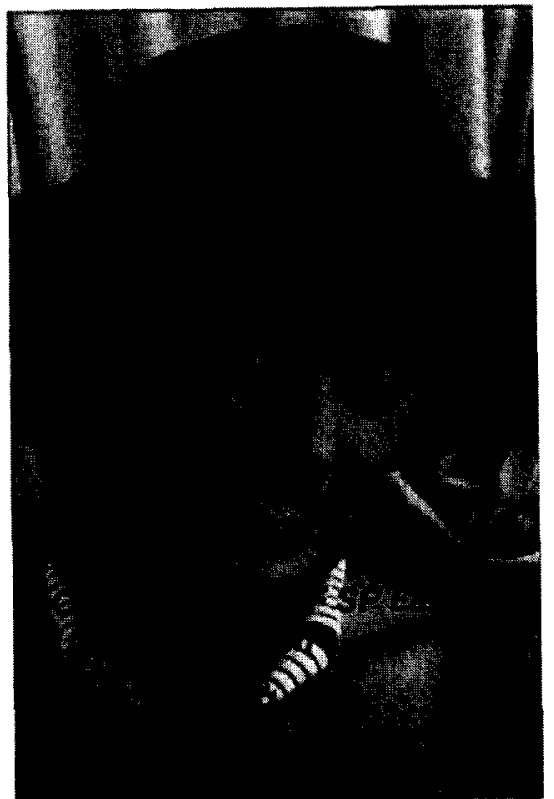

(b)

Fig. 20. Visual Color Image Restoration. (a) Noisy girl; (b) Our restoration.

processing. The test results for a color photo are shown in Fig. 20. It is evident that our Color Visual Group Normalization technique yields excellent contrast and edge-preservation and provides a natural color result for the restored image [48].

\subsection{Imaging enhancement}

Mammograms are complex in appearance and signs of early disease are often small and/or subtle. Digital mammogram image enhancement is particularly important for solving storage and logistics problems, and for the possible development of an automated-detection expert system. The DAF-wavelet based mammogram enhancement is implemented in the following manner. First we generate a perceptual lossless quantization matrix $Q_{j, m}$, to adjust the original transform coefficients $C_{j, m}(k)$. This treatment provides a simple human-vision-based threshold technique for the restoration of the most important perceptual information in an image. For grayscale image contrast stretching, we appropriately normalize the decomposition coefficients according to the length scale, $L$, of the display device [16] so that they fall in the interval of $[0,1]$ of the device frame

$$
N C_{j, m}(k)=Q_{j, m} C_{j, m}(k) / L .
$$

We then use a nonlinear mapping to obtain the desired contrast stretching

$$
\overline{N C_{j, m}}=\gamma_{j, m} X_{j, m}\left(N C_{j, m}\right) \text {, }
$$

where the constant $\gamma_{j, m}$ and function $X_{j, m}$ are appropriately chosen so that the desired portion of the grayscale gradient is stretched or compressed.

To test our new approach, low-contrast and low quality breast mammogram images are employed. A typical low quality front-view image is shown in Fig. 21a. The original image is coded at $512 \times 512$ pixel size with 2 bytes/pixel and 12 bits of gray scale. We have applied our edge enhancement normalization and deviceadapted visual group normalization. As shown in Fig. 21 b, and Fig. $21 \mathrm{c}$, there is a significant improvement in 


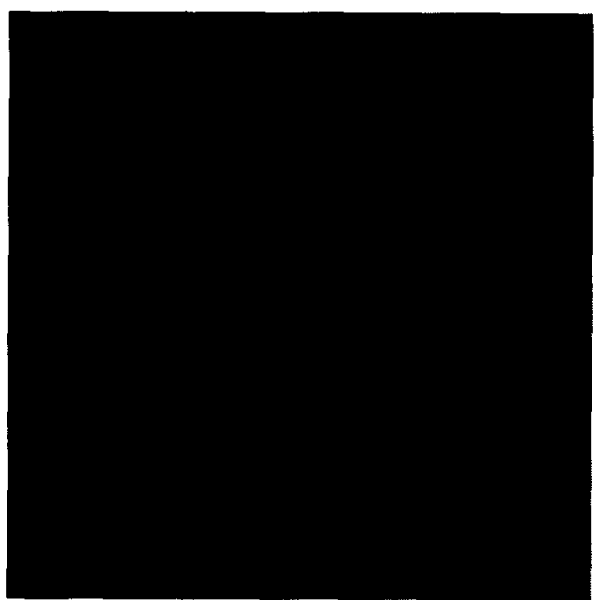

(a)

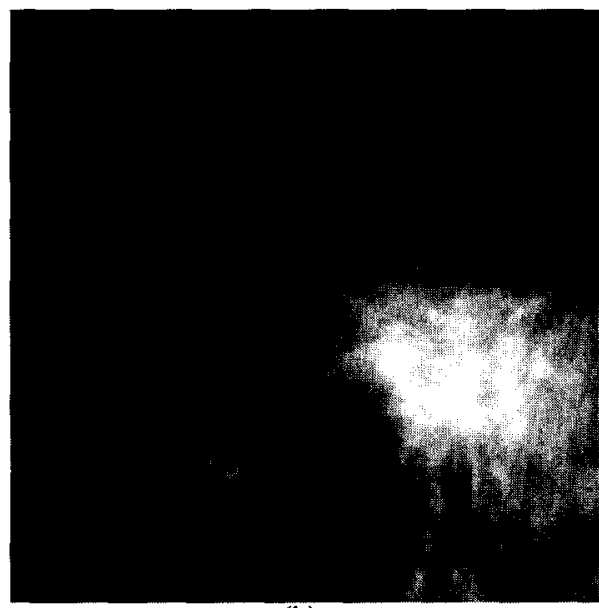

(b)

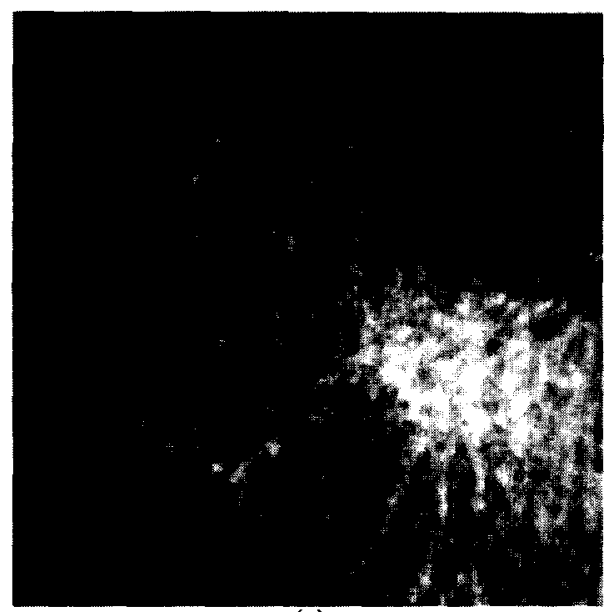

(c)

Fig. 21. Enhancement of Database 1. (a) Original mammogram; (b) Linear enhancement; (c) Nonlinear enhancement.

both the edge representation and image contrast. In particular, the domain and internal structure of high-density cancer tissues are more clearly displayed. Fig. 22a is an original $1024 \times 1024$ side-view breast image which has been digitized to a 200 micron pixel edge with 8 bits of gray scale. The enhanced image result is shown in Fig. 22b. In this case we again obtain a significant improvement in image quality [54,55].

\section{Conclusion}

In summary, in this paper we present a new class of wavelets-generalized symmetric interpolating wavelets (GSIW), which are generated by a window modulated interpolating shell. Due to the absence of a complicated factorization process, this kind of interpolating wavelet is easily implemented and possesses very good characteristics in both time (space) and spectral domains. The stable boundary adjustment can be generated by a window shifting operation only. It overcomes the overshoot of the boundary response introduced by other boundary processing, such as Dubuc Lagrange wavelet and Daubechies boundary filters. Many successful 


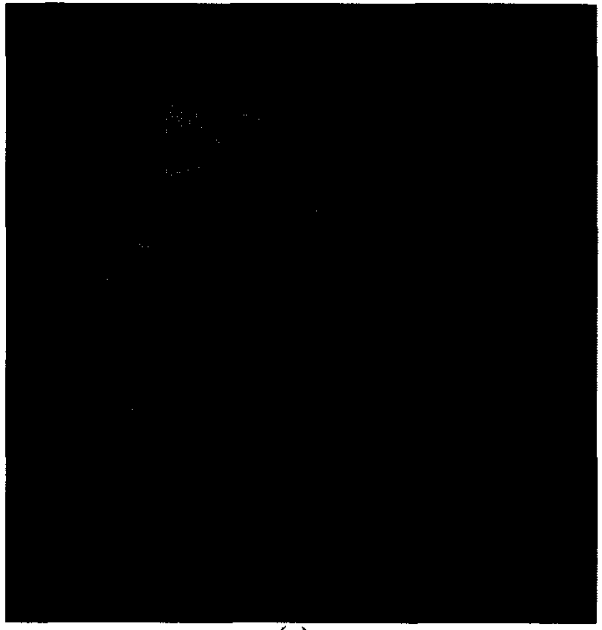

(a)

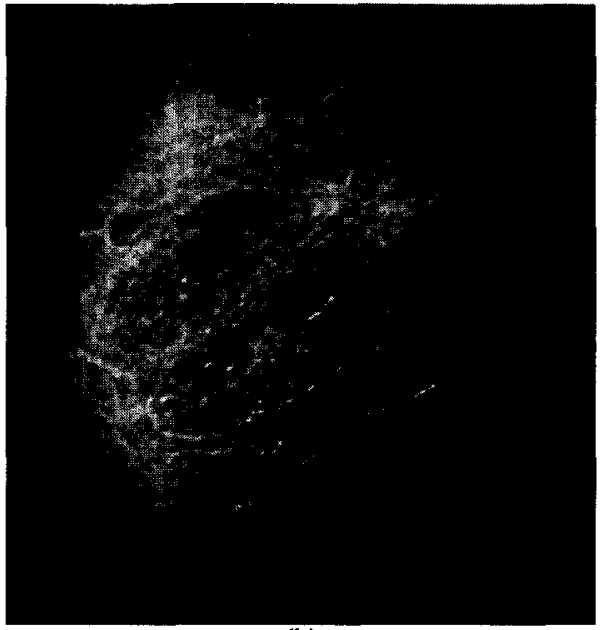

(b)

Fig. 22. Enhancement of Database 2. (a) Original mammogram; (b) Enhancement.

applications of DAF-wavelets have been reported to illustrate its practicality and its mathematical behavior.

\section{References}

[1] R. Ansari, C. Guillemot, J.F. Kaiser, Wavelet construction using Lagrange halfband filters, IEEE Trans. CAS 38 (1991) $1116-1118$.

[2| R. Baraniuk, D. Jones, Signal-dependent time-frequency analysis using a radially Gaussian kernel, Signal Processing 32 (1993) 263-284.

|3| C.M. Brislawn, Preservation of subband symmetry in multirate signal coding, IEEE Trans. Signal Process. 43 (1995) 3046-3050.

14| C.K. Chui, An Introduction to Wavelets (Academic Press, New York, 1992).

15] C.K. Chui, Wavelets: A Tutorial in Wavelet Theory and Applications (Academic Press, New York, 1992).

161 A. Cohen, I. Daubechies, J.C. Feauveau, Biorthogonal bases of compactly supported wavelets, Comm. Pure Appl. Math. 45 (1992) $485-560$.

17| I. Daubechies, Orthonormal bases of compactly supported wavelets, Comm. Pure Appl. Math. 41 ( 1988 ) 909-996.

|8| I. Daubechies, The wavelet transform, time-frequency localization and signal analysis, IEEE Trans. Inform. Theory 36 (1990) 961-1003.

[9] G. Deslauriers, S. Dubuc, Symmetric iterative interpolation processes, Construct. Approx. 5 ( 1989) 49-68.

(10] A.P. Dhawan, E. Le Royer, Mammographic feature enhancement by computerized image processing, Comput. Methods \& Programs Biomed. 27 (1988) 23-35.

[11] D.L. Donoho, De-noising by soft-threshold, IEEE Trans. Inform. Theory 41 (1995) 613-627.

[12] D.L. Donoho, Interpolating wavelet transform, preprint (Stanford Univ., 1992).

[13] S. Dubuc, Interpolation through an iterative scheme, J. Math. Anal. \& Appl. 114 (1986) 185-204.

[14] A. Frishman, D.K. Hoffman, D.J. Kouri, Distributed approximating functional fit of the H3 ab initio potential-energy data of Liu and Siegbahn, J. Chem. Phys. 107 (1997) 804-811.

[15] L. Gagnon, J.M. Lina, B. Goulard, Sharpening enhancement of digitized mammograms with complex symmetric Daubechies wavelets, preprint.

[16] R. Gordon, R.M. Rangayan, Feature enhancement of film mammograms using fixed and adaptive neighborhoods, Appl. Opt. 23 (1984) 560-564.

[17] A. Harten, Multiresolution representation of data: a general framework, SIAM J. Numer. Anal. 33 (1996) $1205-1256$.

[18] C. Herley, M. Vetterli, Orthogonal time-varying filter banks and wavelet packets, IEEE Trans. Signal Process. 42 (1994) $2650-2663$.

[19] C. Herley, Z. Xiong, K. Ramchandran, M.T. Orchard, Joint space-frequency segmentation using balanced wavelet packets trees for least-cost image representation, IEEE Trans. Image Process. 6 (1997) 1213-1230.

[20] D.K. Hoffman, N. Nayar, O.A. Sharafeddin, D.J. Kouri, Analytic banded approximation for the discretized free propagator, J. Phys. Chem. 95 (1991) 8299-8305. 
[21] D.K. Hoffman, A. Frishman, D.J. Kouri, Distributed approximating functional approach to fitting multi-dimentional surfaces, Chem. Phys. Lett. 262 (1996) 393-399.

[22] D.K. Hoffman, G.W. Wei, D.S. Zhang, D.J. Kouri, Shannon-Gabor wavelet distributed approximating functional, Chem. Phys. Lett. 287 (1998) 119-124.

[23] L.C. Jain, N.M. Blachman, P.M. Chapell, Interference suppression by biased nonlinearities, IEEE Trans. Inform. Theory 41 (1995) 496-507.

[24] N. Jayant, J. Johnston, R. Safranek, Signal compression based on models of human perception, Proc. IEEE. 81 (1993) $1385-1422$.

[25] M.A. Kon, L.A. Raphael, Convergence rates of multiscale and wavelet expansions I \& II, manuscript (1998).

[26] J. Kovacevic, M. Vetterli, Perfect reconstruction filter banks with rational sampling factors, IEEE Trans. Signal Process. 41 (1993) $2047-2066$.

[27] J. Kovacevic, W. Swelden, Wavelet families of increasing order in arbitrary dimensions, IEEE Trans. Image Process. (1997), submitted.

[28] S. Lai, X. Li, W.F. Bischof, On techniques for detecting circumscribed masses in mammograms, IEEE Trans. Med. Imag. 8 (1989) 377-386.

[29] A.F. Laine, S. Schuler, J. Fan, W. Huda, Mammographic feature enhancement by multiscale analysis, IEEE Trans. MI 13 (1994) 725-740.

[30] J. Lu, D.M. Healy, Jr., Contrast enhancement via multiscale gradient transform, preprint.

[31] J. Lu, D.M. Healy, Jr., J.B. Weaver, Contrast enhancement of medical images using multiscale edge representation, Opt. Eng., in press.

[32] S. Mallat, A theory for multiresolution signal decomposition: the wavelet representation, IEEE Trans. PAMI 11 (1989) 674-693.

[33] S. Mallat, S. Zhong, Characterization of signals from multiscale edges, IEEE Trans PAMI 14 (1992) 710-732.

[34] Y. Meyer, Wavelets Algorithms and Applications (SIAM, Philadelphia, PA, 1993).

[35] M. Nagao, T. Matsuyama, Edge preserving smoothing, Comput. Graphics \& Image Process. 9 (1979) 394-407.

[36] K. Ramchandran, M. Vetterli, Best wavelet packet bases in a rate-distortion sense. IEEE Trans. Image Process. 2 (1993) $160-175$.

[37] K. Ramchandran, Z. Xiong, K. Asai, M. Vetterli, Adaptive transforms for image coding using spatially-varying wavelet packets, IEEE Trans. Image Process. 5 (1996) 1197-1204.

[38] O. Rioul, M. Vetterli, Wavelet and signal processing, IEEE Signal Process. Mag. 14-38 (October 1991).

[39] A.M. Rohaly, A.J. Ahumada, A.B. Watson, Object detection in natural backgrounds predicted by discrimination performance and models, Vision Research 37 (1997) 3225-3235.

[40] N. Saito, G. Beylkin, Multiscale representations using the auto-correlation functions of compactly supported wavelets, IEEE Trans. Signal Process. 41 (1993) 3584-3590.

[41] A. Scheer, F.R.D. Velasco, A. Rosenfield, Some new image smoothing techniques, IEEE Trans. Syst. Man. Cyber. SMC-10, no. 3 (1980) 153-158.

[42] M.J. Shensa, The discrete wavelet transform: wedding the a trous and Mallat algorithms, IEEE Trans. Signal Process. 40 (1992) 2464-2482.

143] Zhuoer Shi, Zheng Bao, Group-normalized processing of complex wavelet packets, Science China Ser. E 40 (1997) $28-43$.

[44] Z. Shi, Z. Bao, Group-normalized wavelet packet signal processing, Wavelet Application IV, SPIE, Vol. 3078 (1997) pp. $226-239$.

[45] Z. Shi, Z. Bao, Fast image coding of interval interpolating wavelets, Wavelet Application IV, SPIE, Vol. 3078 (1997) pp. $240-253$.

[46] Zhuoer Shi, G.W Wei, D.J. Kouri, D.K. Hoffman, Perceptual image processing using Gaussian-Lagrange distributed approximating functional wavelets, IEEE SP Lett. (1998), submitted.

[47] Zhuoer Shi, G.W. Wei, D.J. Kouri, D.K. Hoffman, Z. Bao, Lagrange wavelets for signal processing, IEEE Trans. Image Process. (1998), submitted.

[48] Zhuoer Shi, G.W. Wei, D.J. Kouri, D.K. Hoffman, Z. Bao, Visual multiresolution color image restoration, IEEE Trans. PAMI (1998), submitted.

[49] Zhuoer Shi, G.W. Wei, D.J. Kouri, D.K. Hoffman, Perceptual normalized subband image restoration, IEEE Symposium on Timefrequency and Time-scale Analysis, N. 144, Pittsburgh, Pennsylvania, Oct. 6-9, 1998.

[50] Zhuoer Shi, Z. Bao, Group normalized wavelet packet transform, IEEE Trans, CAS II (1998), in press.

[51] Zhuoer Shi, Z. Bao, Slow-moving ship target extraction using complex wavelet packet, IEEE Trans. Signal Process. (1998), submitted.

[52] Zhuoer Shi, Z. Bao, L.C. Jiao, Normalized wavelet packet image processing, IEEE Trans. Image Process. (1998), submitted.

[53] Zhuoer Shi, Z. Bao, L.C. Jiao, Wavelet packet based ECG filtering, IEEE Trans. BE (1998), submitted.

[54] Zhuoer Shi, G.W. Wei, D.J Kouri, D.K. Hoffman, Perceptual multiresolution enhancement, IEEE ICASSP (1998), submitted.

[55] Zhuoer Shi, D.J. Kouri, D.K. Hoffman, Mammogram enhancement using generalized Sinc wavelets, IEEE Trans. Med. Imag. (1998), submitted.

156] W. Swelden, The lifting scheme: a custom-design construction of biorthogonal wavelets, Appl. \& Comput. Harmonic Anal. 3 (1996) 186-200.

[57] P.G. Tahoces, J. Correa, M Souto, C. Gonzalez, L. Gomez, J.J. Vidal, Enhancement of chest and breast radiographs by automatic spatial filtering, IEEE Trans. Med. Imag. 10 (1991) 330-335. 
[58] T.D. Tran, R. Safranek, A locally adaptive perceptual masking threshold model for image coding, Proc. ICASSP (1996).

[59] M. Unser, A. Adroubi, M. Eden, The $\mathrm{L}_{2}$ polynomial spline pyramid, IEEE Trans. PAMI 15 ( 1993) 364-379.

[60] P. Vaidyanathan, T. Chen, Role of anti-causal inverse in multirate filter-banks - Part I: system-theoretic fundamentals, IEEE Trans. Signal Process. 43 (1995) 1090-1102.

[61] P. Vaidyanathan, T. Chen, Role of anti-causal inverse in multirate filter-banks - Part II: the FIR case, factorizations, and biorthogonal lapped transforms, IEEE Trans. Signal Process. 43 (1995) 1103-1115.

[62] M. Vetterli, C. Herley, Wavelet and filter banks: theory and design, IEEE Trans. Signal Process. 40 (1992) $2207-2232$.

[63] J.D. Villasenor, B. Belzer, J. Liao, Wavelet filter evaluation for image processing, IEEE Trans. Image Process. 4 (1995) 1053-1060.

[64] A.B. Watson, G.Y. Yang, J.A. Solomon, J. Villasenor, Visibility of wavelet quantization noise, IEEE Trans. Image Process. 6 (1997) $1164-1175$.

[65] G.W. Wei, D.S. Zhang, D.J. Kouri, D.K. Hoffman, Lagrange distributed approximating Functionals, Phys. Rev. Lett. 79 (1997) 775-779.

[66] G.W. Wei, D.J. Kouri, D.K. Hoffman, Wavelets and distributed approximating functionals, Phys. Rev. Lett., submitted.

[67] G.W. Wei, S.C. Althorpe, D.J. Kouri, D.K. Hoffman, An application of distributed approximating functional-wavelets to reactive scattering, J. Chem. Phys. 108 (1998) 7065-7069.

[68] Z. Xiong, K. Ramchandran, M.T. Orchard, Space-frequency Quantization for Wavelet Image Coding, IEEE Trans. Image Process. 6 (1997) 677-693. 